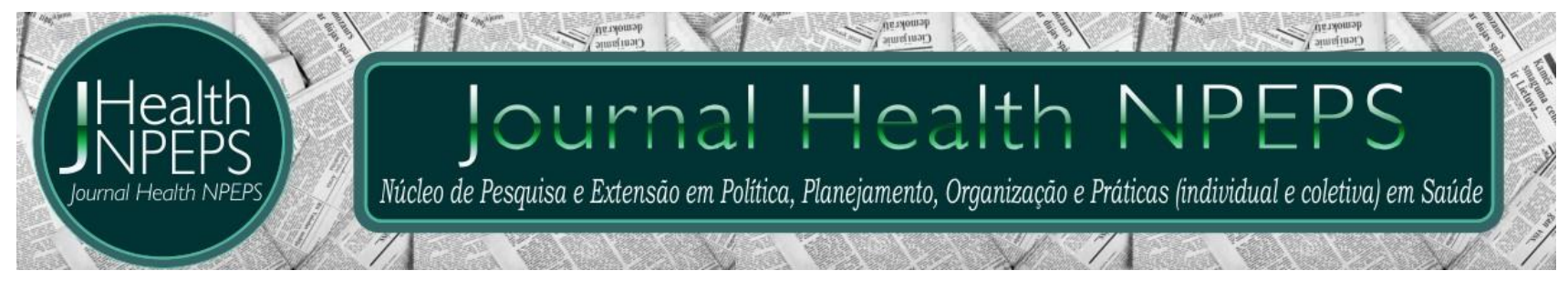

http://dx.doi.org/10.30681/252610104646

ARTIGO ORIGINAL

\title{
Síndrome coronariana aguda associada a teoria do relógio biológico da medicina tradicional chinesa
}

\section{Acute coronary syndrome associated with the biological clock theory of traditional chinese medicine}

\section{Síndrome coronario agudo asociado la teoría del reloj biológico de la medicina tradicional chino}

\section{Eleine Aparecida Penha Martins ${ }^{1}$, Beatriz Maria dos Santos Santiago Ribeiro ${ }^{2}$, Denise Veloso Moreira ${ }^{3}$, Sandra Silverio-lopes ${ }^{4}$, Glaucia de Souza Onori Maier ${ }^{5}$}

\section{RESUMO}

Objetivo: caracterizar o horário do agravo dos pacientes com síndrome coronariana aguda, correlacionando com a teoria do relógio biológico segundo a medicina tradicional chinesa. Método: trata-se de uma pesquisa quantitativa e a coleta de dados foi realizada em um hospital público terciário no Sul do Brasil, por meio de busca ativa diária de todos os pacientes que foram admitidos com diagnóstico de síndrome coronariana aguda. A amostra foi de 94 pacientes, com média de idade de 54 anos, $52,1 \%$ do sexo masculino. Realizou-se entrevista no momento da admissão, busca de informações nos prontuários, comparação dos horários de início e piora da dor, da tomada de decisão para pedir ajuda e da chegada do paciente no hospital. Resultados: o horário com maior incidência de agravos foi entre 21:00h às 23:00h, horário do triplo aquecedor, seguido do horário entre 11:00h às 13:00h, horário do coração. Conclusão: houve aproximação entre o horário de agravo da síndrome coronariana aguda e da teoria do relógio biológico da medicina tradicional chinesa. Esses achados apontam novos conhecimentos para a enfermagem cardiológica e o planejamento da assistência.

Descritores: Acupuntura; Doença das Coronárias; Enfermagem.

\footnotetext{
${ }^{1}$ Enfermeira. Doutora em Enfermagem. Docente de Enfermagem da Universidade Estadual de Londrina (UEL). Londrina, Paraná, Brasil. E-mail: eleinemartins@gmail.com ORCID ID: https://orcid.org/0000-0001-6649-9340

${ }^{2}$ Enfermeira. Mestre em Enfermagem. Doutoranda de Enfermagem pela Universidade de São Paulo (USP). Ribeirão Preto, São Paulo, Brasil. E-mail: beatrizsantiago1994@hotmail.com ORCID ID: https://orcid.org/0000-0001-5211-5422 Autor para correspondência - Endereço: Rua: Angélica Thaís Fraga, n43, Jardim Violeta- Califórnia- Paraná.

${ }^{3}$ Educadora Física. Mestre em Tecnologia em Saúde. Auxiliar de Coordenação da Pós-graduação em Acupuntura. Curitiba, Paraná, Brasil. E-mail: monografiasacup@ibrate.edu.br ORCID ID: https://orcid.org/0000-0002-4907-8170

${ }^{4}$ Fisioterapeuta. Doutora em Ciências dos Desportos. Coordenadora de pós-graduação em Acupuntura, Faculdade de Tecnologia IBRATE. Curitiba, Paraná, Brasil. E-mail: san.silverio@yahoo.com.br ORCID ID: https://orcid.org/00000002-5424-6140

${ }^{5}$ Enfermeira. Mestra em Enfermagem. Enfermeira na hemodinâmica do Hospital Universitário de Londrina. Londrina, Paraná, Brasil. E-mail: glauciaomori@yahoo.com.br ORCID ID: https://orcid.org/0000-0003-4663-0190
}

Este artigo está licenciado sob forma de uma licença Creative Commons Atribuição 4.0 Internacional, que permite uso irrestrito, distribuição e reprodução em qualquer meio, desde que a publicação original seja corretamente citada 


\begin{abstract}
Objective: to characterize the time of illness in patients with acute coronary syndrome, correlating with the biological clock theory according to traditional Chinese medicine. Method: this is a quantitative research and data collection was carried out in a public tertiary hospital in southern Brazil through an active daily search of all patients who were admitted with a diagnosis of acute coronary syndrome. The sample consisted of 94 patients, with a mean age of 54 years, 52.1\% male. Interview at the time of admission, search for information in medical records, comparison between the time of onset and worsening of pain, decision making to ask for help and the patient's arrival at the hospital were carried out. Results: the highest incidence of injuries was observed during the time of the triple heater between 9 and $11 \mathrm{pm}$, followed by the heart time between 11 am and $1 \mathrm{pm}$. Conclusion: there was an approximation between the time of aggravation of the acute coronary syndrome and the biological clock theory of traditional Chinese medicine. These findings point to new knowledge for cardiological nursing and care planning.

Descriptors: Acupuncture; Coronary Disease; Nursing.
\end{abstract}

\title{
RESUMEN
}

Objetivo: caracterizar el tiempo de enfermedad en pacientes con síndrome coronario agudo, correlacionando con la teoría del reloj biológico según la medicina tradicional china. Método: se trata de un investigacion cuantitativo, la recogida de datos se realizó en un hospital terciario público del sur de Brasil, mediante una búsqueda activa diaria de todos los pacientes que ingresaron con diagnóstico de síndrome coronario agudo. La muestra estuvo formada por 94 pacientes, con una edad media de 54 años, 52,1\% varones. Se realizó entrevista en el momento del ingreso, búsqueda de información en la historia clínica, comparación de los tiempos de inicio y agravamiento del dolor, toma de decisiones para pedir ayuda y llegada del paciente al hospital. Resultados: la hora con mayor incidencia de lesiones fue entre las 21:00 a 23:00, hora del triple calentador, seguida por la hora entre las 11:00 a 13:00, hora del corazón. Conclusión: hubo una aproximación entre el tiempo de agravamiento del síndrome coronario agudo y la teoría del reloj biológico de la medicina tradicional china. Estos hallazgos apuntan a nuevos conocimientos para la enfermería cardiológica y la planificación del cuidado.

Descriptores: Acupuntura; Enfermedad Coronaria; Enfermería.

\section{INTRODUÇÃO}

Há alguns anos, as doenças cardiovasculares (DCV) ocupam lugar de destaque entre as principais causas de morte no mundo. Em 2017, no Brasil, houveram 383.961 mortes por DCV. A Sociedade Brasileira de Cardiologia
contabiliza que somente de janeiro
ao final de junho de 2020 foram registradas mais de 200.000 mortes por DCV no território brasileiro. O principal representante das Doenças Isquêmicas do Coração é o Infarto Agurdo do Miorcádio (IAM) ${ }^{1}$.

O objetivo em comum entre a medicina ocidental, medicina tradicional chinesa (MTC) e a psicossomática é que esses três conhecimentos procuram estabelecer o bem-estar do paciente e 
consideram a saúde como o estado mais precioso do ser humano. A MTC se preocupa em identificar o desequilíbrio energético dos órgãos e vísceras antes que se instale a doença². A psicossomática trata o sintoma da doença como uma repercussão da alma. Nessa pesquisa, há o interesse na interface desses três conhecimentos, porém a ênfase será direcionada à $M^{2} C^{3}$.

$\mathrm{Na}$ MTC, a doença se instala quando há um desequilíbrio entre as energias provindas da natureza externa (frio, vento, calor de verão, umidade, secura) e o mecanismo de resistência do corpo. Porém, também considera que o desequilíbrio das emoções (excitação/alegria, raiva, tristeza, medos, preocupações) afetam os órgãos ${ }^{3,4}$.

Cada elemento tem suas funções em evidência em algum período do dia ou do ano, podendo ser caracterizado pelo ciclo sazonal, sequência cosmológica, geração, controle, super atuação e de afrontação ${ }^{3}$. Entre esses ensinamentos, há algumas teorias como a dos cinco elementos que dividem os fenômenos e o ser humano em cinco classificações: fogo, água, madeira, terra e metal, bem como as horas do dia, com divisão em 12 intervalos de duas horas cada, constituindo, portanto, a teoria do relógio biológico ${ }^{3}$.

Segundo tal teoria, a cada duas horas, um dos meridianos de energia concentraria a maior parte de energia vital circulante como se os demais meridianos "emprestassem" um pouco da sua própria energia, fazendo com que o meridiano do horário se enchesse mais dela. Isso daria a força para que o órgão ou víscera correspondente àquele meridiano pudesse cumprir mais adequadamente as funções fisiológicas do corpo. Saber se tem um horário em que os sintomas se agravam pode auxiliar na orientação do diagnóstico e do tratamento 3 .

As principais funções do coração na MTC é a de governar o sangue, os vasos sanguíneos e abrigar a mente. Dentro dessa atividade, significa que o estado do coração afetará as atividades mentais, incluindo o estado emocional. No seu desequilíbrio podem ocorrer problemas mentais/emocionais, memória pobre, pensamento obscuro, insônia ou sonolência até a inconsciência ${ }^{3}$.

O coração é um órgão vital capaz de gerar e sustentar a vida. A sua falência leva a algumas arritmias, as quais requerem um tratamento específico de acordo com a causa. A situação extrema a qual necessita de 
atenção imediata é a parada cardíaca que, apesar de ser bem atendida, pode ocorrer danos irreparáveis ao organismo podendo deixar sequelas e até mesmo levar a óbito1. Na MTC, o coração também é considerado um dos mais importantes órgãos internos, sendo chamado de "soberano, imperador ou monarca"3.

$\mathrm{Na}$ síndrome coronariana aguda (SCA), o paciente apresenta evidências clínicas e/ou laboratoriais de isquemia miocárdica aguda produzida por desequilíbrio entre oferta e demanda de oxigênio para o miocárdio, tendo como causa principal a instabilidade de uma placa aterosclerótica. Constitui-se, assim, um grupo de sintomas que inclui dor torácica, desconforto difuso retroesternal e, em alguns casos, irradiase para braço esquerdo e/ou direito, ombros, pescoço ou mandíbula, dor epigástrica, sudorese, mal-estar, indigestão, entre outros, cuja incidência apresenta-se no Brasil na ordem de causas de morbimortalidade nas últimas décadas ${ }^{5}$.

Somando a essas informações, a MTC também tece que cada órgão tem o seu próprio relógio biológico dentro das 24 horas, ou seja, é o momento em que o órgão atua com toda energia. Nesse caso, o coração tem sua ação máxima das $11: 00$ h̀s $13: 00 h^{6}$.

Dentro da linguagem da fisiologia e medicina praticada no ocidente, a influência do horário do dia, no ritmo do corpo e da saúde, é conhecido como ciclo circadiano. Os ritmos circadianos são mediados pelo sistema nervoso central e também no interior das células periféricas, onde diversas atividades diárias são influenciadas, abrangendo o sono, o tempo de alimentação, o metabolismo energético, funções endócrinas e imunológicas ${ }^{7}$.

A MTC vem sendo explorada por todo seu alcance clínico e comprovações científicas $^{8}$ por vários profissionais, principalmente os da área de enfermagem ${ }^{9}$. Todavia, há necessidade de mais estudos que revelem as particularidades dessa prática na SCA.

Nesse sentido, surge-se a seguinte pergunta de pesquisa: os pacientes buscam ajuda/atendimento médico pré-hospitalar ou intra-hospitalar no horário de governabilidade energética do coração, segundo a MTC ou no horário em que a dor fica insuportável? A partir disso, o estudo teve como objetivo caracterizar o horário do agravo dos pacientes com SCA, correlacionando com a teoria do horário biológico, segundo a MTC. 


\section{MÉTODO}

Trata-se de pesquisa quantitativa exploratória, sendo selecionados pacientes maiores de 18 anos que concordaram em participar da pesquisa, assinando o termo de consentimento livre e esclarecido, admitidos neste serviço com diagnóstico de SCA (angina instável, infarto agudo do miocárdio com ou sem supra-desnivelamento do segmento ST), confirmado durante o período de internação por diagnóstico médico. Foram excluídos os pacientes que entraram com dor torácica e, posteriormente, tiveram diagnóstico diferente ao almejado no presente estudo.

A pesquisa foi realizada em um hospital terciário do Sul do Brasil com 94 clientes diagnosticados com SCA. Essa instituição possui hemodinâmica e atende cardiologia, ortopedia, vascular e neurocirurgia. A hemodinâmica desse serviço no período de coleta tinha fluxo anual aproximado de 260 cineangiocoronariografias aproximadamente 50 angioplastias coronárias por ano. 0 pronto socorro dessa intituição possui 48 leitos, sendo três destinados a emergência e 45 leitos de internação. Segundo o relatório de serviço de atendimento desse hospital, há em média 8 mil internações/ano, possui taxas de ocupação de $100 \%$ dos seus leitos e um fluxo de três mil atendimentos em média, por mês.

A coleta de dados ocorreu no período de outubro de 2012 a abril de 2013. Foi realizada entrevista no momento da admissão, busca de informações nos prontuários, comparação dos horários de início e piora da dor, da tomada de decisão para pedir ajuda e da chegada do paciente no hospital. Assim, após a inserção do paciente nesse estudo, os dados foram coletados por entrevista, sendo complementadas por informações do prontuário do paciente e também utilizou um formulário composto por seis campos: caracterização sóciodemográfica, fatores predisponentes e antecedentes pessoais dos entrevistados, antecedentes familiares, história atual da doença, cálculo do escore de Thrombolysis in Myocardial Infarction (TIMI) e Global Registry of Acute Coronary Events (GRACE) e, o último campo, com o tratamento ao qual foram submetidos e os desfechos hospitalares dos pacientes do estudo.

O escore de GRACE fornece uma ideia da extensão do dano miocárdico, avalia o risco de mortalidade dentro do 
hospital e após seis meses de alta. A pontuação do escore até 108 pontos classifica os pacientes de baixo risco, indicando mortalidade hospitalar inferior a 1\%. Pontuações entre 109 e 140 são os pacientes com risco intermediário, sendo o risco de mortalidade de 1 a $3 \%$, já as pontuações maiores que 140 apresentam risco alto para mortalidade, podendo alcançar os $3 \%$. E o escore de TIMI, considera as variáveis da apresentação clínica da SCA como alteração do segmento ST, elevação de marcador de necrose miocárdica, mais de um episódio de angina em 24 horas ou as características prévias dos pacientes, como idade maior ou igual a 65 anos, uso de aspirina, obstrução coronária superior a $50 \%$ e mais de três fatores de risco para doença aterosclerótica. A pontuação mínima pelo escore TIMI é zero, e a máxima 14 , sendo baixo risco pontuação entre 0 e 2; médio risco pontuação de 3 a 5; e alto risco pontuação maior que $5^{10}$.

As estações do ano foram categorizadas conforme os dados do Instituto Nacional de Meteorologia $(\text { INMET })^{11}$. E na MTC, o verão é representado pelo fogo; a madeira pela primavera; o outono pelo metal e o inverno pela água ${ }^{3}$.
Os dados foram tabulados em programa Microsoft $₫$ excel 2010 e, posteriormente, realizado a análise estatística descritiva (frequência absoluta e relativa), aproximando os dados com a teoria do relógio biológico.

No que tange aos aspectos éticos foi firmado o termo de sigilo e confidencialidade com a instituição de origem dos pacientes, sendo um compromisso de responsabilidade sobre todas as informações técnicas e outras relacionadas a coleta dos dados da pesquisa. 0 projeto desse estudo foi apreciado e aprovado pelo Comitê de Ética em Pesquisa com Seres Humanos da Universidade Estadual de Londrina (UEL), pelo CAAE $\mathrm{n}^{\circ}$ 07063312.7.0000-5231 e parecer n. 139.509.

\section{RESULTADOS}

Participaram do estudo 94 pacientes. No que tange os antecedentes pessoais, os fatores de risco prevalentes foram 0 sedentarismo (85,2\%), hipertensão (72,3\%), com histórico familiar: dislipidemia $(52,1 \%)$, estresse $(50,0 \%)$, diabetes $(53,2 \%)$, tabagismo $(31,9 \%)$, obesidade $(30,8 \%)$ e etilismo $(8,5 \%)$, sendo a hipertensão prevalente no sexo feminino. Os antecedentes familiares comuns foram a hipertensão 
arterial (33\%), o infarto agudo (50\%), foram considerados alterados diabetes $(20 \%)$ e AVC $(20 \%)$. No que se condizentes ao quadro de SCA. refere aos escores de TIMI e GRACE,

Quadro 1 - Distribuição dos diagnósticos clínicos dos pacientes do estudo.

\begin{tabular}{|l|c|}
\hline \multicolumn{1}{|c|}{ Diagnóstico clínico } & Incidência percentual \\
\hline Angina Instável & $39,4 \%$ \\
\hline Infarto com supradesnivelamento do segmento ST & $34,0 \%$ \\
\hline Infarto sem supradesnivelamento do segmento ST & $26,6 \%$ \\
\hline
\end{tabular}

$\mathrm{Na}$ admissão nesse hospital público terciário, os pacientes foram categorizados como escores de risco no TIMI e Grace. A estratificação de risco na admissão identificou que $80,9 \%$ dos pacientes tinham de 0 a 4 pontos (TIMI) e 74,5\% até 140 pontos (GRACE). A média de idade foi de 54 anos, com predomínio do sexo masculino $(52,1 \%)$.
Em relação a faixa etária, prevaleceu maiores de 50 anos (78,7\%).

A cor predominante foi a branca com 55,3\%, seguida da negra e parda, ambas com 22,3\%. Desses, 46,9\% estudaram até quatro anos, 59,5\% não trabalhavam na época da coleta dos dados e 74,4\% recebiam até dois salários mínimos. No que concerne ao estado civil, a maiora eram casados.

Quadro 2 - Distribuição percentual da incidência dos intervalos de horário do relógio biológico, seus meridianos/órgãos/vísceras correspondentes nos momentos de início da dor, piora da dor, pedido de ajuda e chegada no hospital.

\begin{tabular}{|c|c|c|c|c|c|c|}
\hline $\begin{array}{c}\text { Intervalo de } \\
\text { horário }\end{array}$ & Meridiano & $\begin{array}{c}\text { Fase } \\
\text { incidência } \\
\text { Início da dor } \\
(\%)\end{array}$ & $\begin{array}{c}\text { Fase } \\
\text { incidência } \\
\text { Piora da dor } \\
(\%)\end{array}$ & $\begin{array}{c}\text { Fase } \\
\text { incidência } \\
\text { Pedir ajuda } \\
(\%)\end{array}$ & $\begin{array}{c}\text { Fase } \\
\text { incidência } \\
\text { Chegada no } \\
\text { hospital(\%) }\end{array}$ & $\begin{array}{c}\text { Somatória da } \\
\text { incidência } \\
\text { percentual } \\
\text { de todas as } \\
\text { fases }\end{array}$ \\
\hline $23: 00$ às $01: 00 \mathrm{~h}$ & $\mathrm{VB}$ & 14,20 & 5 & - & 14,06 & 28,26 \\
\hline $1: 00$ às 3:00 h & $\mathrm{F}$ & 10,50 & 10 & 4,30 & 12,7 & 37,50 \\
\hline $3: 00$ às $5: 00 \mathrm{~h}$ & $\mathrm{P}$ & 3,51 & 5 & 4,30 & 11,7 & 24,51 \\
\hline $5: 00$ às $7: 00 \mathrm{~h}$ & $\mathrm{IG}$ & 3,51 & 10 & 4,30 & 5,36 & 23,17 \\
\hline $7: 00$ às $9: 00 \mathrm{~h}$ & $\mathrm{E}$ & 4,76 & 10 & 7,65 & - & 22,41 \\
\hline $9: 00$ às $11: 00 \mathrm{~h}$ & $\mathrm{BP}$ & 3,51 & 5 & 7,65 & 11,36 & 27,52 \\
\hline $11: 00$ às $13: 00 \mathrm{~h}$ & $\mathrm{C}$ & 10,50 & 10 & 24,50 & 3.96 & 48,96 \\
\hline $13: 00$ às $15: 00 \mathrm{~h}$ & $\mathrm{ID}$ & 4,76 & 10 & 7,65 & 5,38 & 27,79 \\
\hline $15: 00$ às $17: 00 \mathrm{~h}$ & $\mathrm{~B}$ & 14,25 & 10 & 7,65 & 11,57 & 43,47 \\
\hline $17: 00$ às $19: 00 \mathrm{~h}$ & $\mathrm{R}$ & 10,50 & 11 & - & 7.86 & 29,36 \\
\hline $19: 00$ às $21: 00 \mathrm{~h}$ & PC & 10,50 & 4 & 7,50 & 11,47 & 33,47 \\
\hline $21: 00$ às $23: 00 \mathrm{~h}$ & TA & 9,50 & 10 & 24,50 & 16,4 & 60,40 \\
\hline
\end{tabular}

Legenda: vesícula biliar(VB), fígado(F), pulmão(P), intestino grosso(IG), estômago(E), baçopâncreas(BP), coração(C), bexiga(B), rim (R), pericárdio(PC), triplo aquecedor(TA). 
Conforme o Quadro 2, o horário com maior incidência das pioras estão entre $21: 00 \mathrm{~h}$ às $23: 00 \mathrm{~h} \quad(60,40 \%)$, correspondente na teoria do relógio biológico ao Triplo Aquecedor, seguido do horário do Coração entre 11:00h às 13:00h $(48,96 \%)$.

O início da dor apareceu com maior incidência no horário da Bexiga, que é entre $15: 00 \mathrm{~h}$ às $17: 00 \mathrm{~h}(14,25 \%)$. $\mathrm{Na}$ fase de piora da dor, houve um predomínio do horário do Rim que é entre 17:00h às 19:00h (11\%), seguido de uma pulverização de outros sete órgãos/meridianos, com 10\% cada.

$\mathrm{Na}$ fase de pedir ajuda, ocorreu um importante destaque de incidência de agravo no horário correspondente ao Coração das $11: 00$ h̀s $13: 00 h$ (24,50 \%) e do Triplo Aquecedor das $21: 00 \mathrm{~h}$ às 23:00h $(24,50 \%)$. Para a fase de pedido até a chegada no hospital a incidência maior foi para o horário das $21: 00 \mathrm{~h}$ às 23:00h com $16,04 \%$ correspondente ao Triplo Aquecedor.

Para a composição da Figura 1, agrupou-se os meridianos/órgão/víscera correspondente a cada elemento, a saber: Madeira (VB e F) das 23:00h às 3:00h; Metal ( $P$ e IG) das 3:00h às 7:00h ; Terra (E e BP) das 7:00h às 11:00h; Fogo (C e ID) das 11:00h às 15:00h (TA e PC) das 19:00h às 23:00h; Água (B e R) das 15:00h às 19:00h.

Figura 1 - Percentual da aproximação entre os cinco elementos da MTC, baseado no horário de disparo e agravo da dor, pedido de ajuda e entrada no hospital.

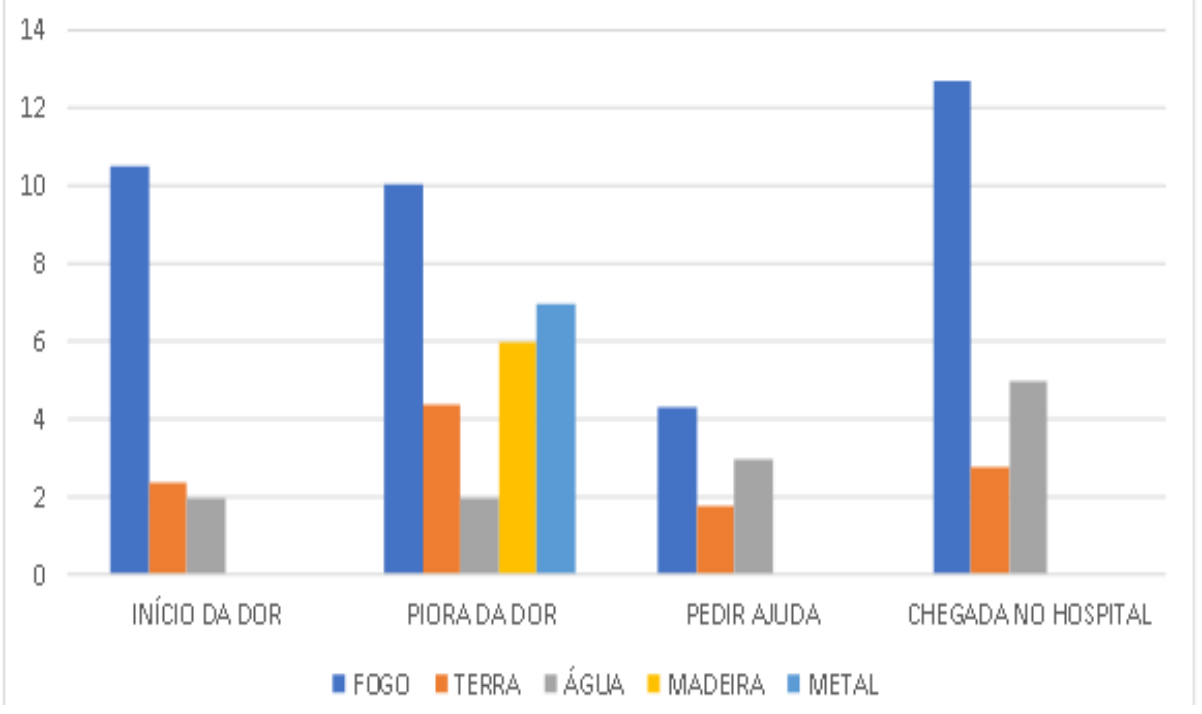

Em todas as fases de agravo da SCA, o elemento fogo representado por Coração (C) e Triplo Aquecedor (TA) apresentaram maior incidência, resultando a média de $15 \%$, no horário das 11:00h às 15:00h. 


\section{DISCUSSÃO}

Há poucos estudos que correlacionam ou dimensionam as patologias da medicina ocidental com o atendimento prestado pela medicina oriental, neste estudo buscou a aproximação entre os dois conhecimentos.

Os dados do perfil epidemiológico para o diagnóstico de SCA condiz com outra pesquisa em uma unidade de clínica médica no Distrito Federal $(B R)^{12}$ e na $\mathrm{China}^{13}$, relacionado ao sexo masculino. Em relação a faixa etária, o presente estudo apresentou média de 54 anos, contrário a estudo realizado em Aracajú (SE), em que prevaleceu pacientes com a média de $63,15 \operatorname{anos}^{14}$ e igualmente a um estudo multicêntrico com 3745 paciente com SCA, em que a média foi de 60,3 anos no sexo masculino e 64,6 no feminino ${ }^{15}$.

Na MTC, a acupuntura tem como representação o zang para os órgãos e o fu para as vísceras, também sendo representados pelo yin e yang, respectivamente ${ }^{3}$. Dos pacientes estudados, o início da dor tanto no sexo feminino como masculino foi em horários da energia yang representada pelo fu, ou seja, energia das vísceras seguida de órgãos. O horário de piora da dor também foi identificado em $45 \%$ dos participantes coincidindo com o horário dos órgãos (Yin) e 55\% em horário das vísceras (Yang) e o momento de pedir ajuda se equilibra entre órgãos e vísceras, ou seja, energia Yin (Coração) e Yang (Triplo Aquecedor). O horário da chegada no pronto socorro ocorreu com maior incidência ao horário de atuação das vísceras (Yang, Fu) ${ }^{3}$.

Um fato que chama atenção é que, muitas vezes, a dor inicia e leva um tempo maior para a chegada do paciente ao hospital. A tomada de decisão para essa busca de atendimento depende dos valores, conhecimentos, crenças e as condições de acesso, partindo do princípio das singularidades individuais e fatores vinculados ao sistema de saúde local ${ }^{16-18}$.

Nesse estudo, o tempo entre o início da dor e o pedido de ajuda demorou de 1022 minutos até 21600 minutos. 0 início da dor começou nas vísceras (bexiga e vesícula biliar) e o momento de pedido de ajuda no meridiano do coração e triplo aquecedor. Os sinais e sintomas deste desequilíbrio energético ou doença podem se apresentar de formas diversas.

Dentro do fluxo das energias e conexões dos meridianos, a energia flui na sequência de vísceras para os órgãos 
e, naturalmente, de órgãos para as vísceras, formando um ciclo, ou seja, da vesícula biliar para o fígado, do pulmão para o intestino grosso, do estômago para o baço pâncreas, do coração para o intestino delgado, da bexiga para os rins, do pericárdio para o triplo aquecedor e o reinício do ciclo $^{19}$. Identificou-se nesse estudo que, na fase da piora da dor, houve manifestação da energia dos rins em $11 \%$ dos pacientes, porém, dentro do fluxo das energias $41 \%$ dos pacientes tiveram o horário de piora desde o meridiano do coração até os rins. Esse fato pode se relacionar com a decisão de pedir ajuda, conforme a manifestação dos sinais e sintomas, e mostra a alteração no fluxo energético.

Há muitos pacientes que sentem a dor precordial, não a valoriza e somente pedem auxílio quando não suportam mais permanecer nesta condição. Embora a dor torácica apareça como principal sintoma da SCA, muitas vezes, pacientes e familiares desconhecem sua sintomatologia, o que contribui para o retardo na procura por atendimento ${ }^{17}$. Outros pacientes sentem a dor e imediatamente solicitam auxílio, principalmente para o serviço préhospitalar ${ }^{18}$. 0 presente estudo vai ao encontro, pois as maiores incidências para o pedido de ajuda foram nos horários entre às 11:00h e 13:00h (meridiano do coração) e entre às $21: 00 \mathrm{~h}$ e 23:00 h (meridiano do triplo aquecedor).

Identifica-se nesse estudo que o horário do início da dor, o horário do encaminhamento ao hospital e o horário de chegada ao hospital estão dentro do meridiano do elemento fogo que acomoda o meridiano do coração/intestino grosso e do pericárdio/triplo aquecedor ${ }^{19}$. Segundo a MTC, a emoção que predomina nos indivíduos quando estão em desequilíbrio dos meridianos e/ou órgão coração (xin) é a euforia, ansiedade, mania ou hiperexcitabilidade ${ }^{20}$. Esse perfil concorda com o que a medicina no ocidente chama de estressado, o qual inclui ansiedade e nervosismo, sendo muito frequente nos cardiopatas ${ }^{20}$.

O elemento predominante na maioria dos casos, foi o fogo, os meridianos/órgãos envolvidos em maior escala foram o coração e o triplo aquecedor. Em concordância aos achados dessa pesquisa, o horário das $11: 00 \mathrm{~h}$ às $13: 00 \mathrm{~h}$ corresponde ao período de maior atividade do coração quando comparado ao relógio biológico utilizado na $M T C^{3,5,6}$.

Estudo realizado na Espanha ${ }^{21}$ sobre a influência do ritmo circadiano na 
deflagração do IAM, concluiu que há um pico de incidência desse agravo por volta das 10:00h da manhã. Esse mesmo estudo detectou que patologias associadas do tipo diabetes e tabagismo, podem influenciar o padrão de horário da incidência de tal patologia. Em Cuba ${ }^{22}$, os horários de apresentação dos sintomas de IAM mais frequentes foram entre as 06:00h e 11:59h, não encontrando diferenças entre os sexos e grupos etários analisados.

A fato de que na fase "entrada no hospital" os percentuais de incidência dos elementos correspondentes aos horários se diluem, não se concentrando em um elemento específico como nas fases anteriores, pode sugerir que 0 paciente com queixas, muitas vezes, organiza-se conforme suas possibilidades e não por urgência, comparecendo no ambiente hospitalar quando alguém pode acompanhá-lo ou levá-lo, pelo seu histórico e nível de conhecimento sobre a gravidade ou ainda por possuir outras retaguardas assistenciais, que o assistem diariamente.

Acredita-se que os achados deste estudo possam auxiliar a enfermagem, principalmente na área cardiológica, a planejar e organizar o serviço quanto ao acolhimento e classificação de risco do paciente com SCA, em especial nos horários de maior incidência e agravo da patologia, e em aspectos educativos/preventivos nos horários de maior incidência podem ser divulgados à população de risco. Não menos importante, o enfermeiro deve conhecer todo processo fisiológico que envolve a dor nos casos de SCA, para tomada de decisão e intervenções rápidas e eficazes, o que envolve também, treinamento e sintonia da equipe ${ }^{23,24}$.

Como limitação do presente estudo, não avaliou a associação com os aspectos sociodemográficos, condições pregressas de saúde, histórico anterior de IAM, marcadores relacionados a inflamação, e influência de outras comorbidades no horário de agravo da SCA. Além disso, na amostra foram incluídos pacientes com diferentes gravidades (angina instável e infarto com e sem supradesnivelamento do segmento ST), o que pode ter causado um viés de observação.

\section{CONCLUSÃO}

A SCA ocorreu tanto em homens como em mulheres jovens, sendo que o início da dor apareceu com maior incidência no horário da bexiga. $\mathrm{Na}$ fase de piora da dor, houve um predomínio do horário do rim. $\mathrm{Na}$ fase de pedir 
ajuda, ocorreu um importante destaque de incidência de agravo no horário correspondente ao coração e ao triplo aquecedor. Para a fase de pedido até a chegada no hospital a incidência maior foi correspondente ao triplo aquecedor.

As informações apresentadas mostram a importância da integração da assistência ao paciente com SCA e o conhecimento da MTC, tanto para apoio como para fins preventivos e organização no ambiente de enfermagem cardiológica. Esse estudo tende a subsidiar novas pesquisas relacionadas à SCA, para maior aproximação do conhecimento da MTC e a medicina ocidental.

\section{REFERÊNCIAS}

1. Silva LCMA, Silva TTM, Costas ICS, Lima MSM, Dantas DV, Dantas RAN. Aspectos epidemiológicos, clínicos e angiográficos de pacientes submetidos a intervenção coronária percutânea primária. Ciênc cuid saúde. 2020; 19:e47408.

2. Cintra MER, Pereira PPG. Percepções de corpo identificadas entre pacientes e profissionais de medicina tradicional chinesa do centro de saúde escola do Butantã. Rev saúde soc. 2012; 21(1):193-205.
3. Macioccia G. Os fundamentos da medicina chinesa: um texto abrangente paraacupunturistas e fitoterapeutas. São Paulo: Editora Roca; 2007

4. Wembu X. Tratado de medicina chinesa. São Paulo: Editora Roca; 2011.

5. Diretriz de Telecardiologia no Cuidado de Pacientes com Síndrome Coronariana Aguda e Outras Doenças Cardíacas. Arq Bras Cardiol. 2015; 104(5);1-26.

6. Requena Y. Acuputura e Psicologia. São Paulo: Editora Andrei; 1990.

7. Cutolo M. Circadian rhythms and rheumatoid arthritis. Joint Bone Spine-Revue du Rumatisme. 2019; 86(3):327-333.

8. Silvério-Lopes S. Analgesia por acupuntura. Curitiba: Editora Omnipax; 2013.

9. Mendes DS, Moraes FS, Lima GO, Silva PR, Cunha TA, Crossetti MGO, et al. Benefícios das práticas integrativas e complementares no cuidado de enfermagem. J Health NPEPS. 2019; 4(1):302-318.

10. Correia LCL, Freitas R, Bittencourt AP, Souza AC, Almeida MC, Leal J, et al. Valor prognóstico do Escore de Risco GRACE versus Escore de Risco TIMI em síndromes coronarianas 
agudas. Arq Bras Cardiol. 2010; 94(5):613-619.

11. Brasil. Instituto Nacional de Meteorologia. Ministério da agricultura, pecuaria e abastamento. Brasília: INMET; 2016.

12. Silva AJS, Guimarães CSS, Reis JA. Perfil de pacientes internados com diagnóstico de síndrome coronariana. Rev Soc Bras Clin Med. 2018; 16(2):104-107.

13. Yun-Peng K, Li-Ying C, Tie-Duo K, Wen-Xian L. Características Clínicas e Eventos Adversos em Pacientes com Síndrome Coronariana Aguda e História de Doença Arterial Periférica. Arq Bras Cardiol. 2019; 113(3):367-372.

14. Oliveira LMSM, Costa IMNBC, Silva DG, Silva JRSS, Barreto-Filho JAS, Almeida-Santos MA. Reinternação de Pacientes com Síndrome Coronariana Aguda e seus Determinantes. Arq Bras Cardiol. 2019; 113(1):42-49.

15. Soeiro AM, Silva PGMB, Roque EAC, Bossa AS, Biselli B, Leal TCAT, et al. Diferenças Prognósticas entre Homens e Mulheres com Síndrome Coronariana Aguda. Dados de um Registro Brasileiro. Arq Bras Cardiol. 2018; 111(5):648-653.

16. Brasil. Conselho Nacional de Secretários de Saúde (Conass). Rede de atenção às urgências e emergências: avaliação da implantação e do desempenho das unidades de pronto atendimento. Brasília: CONASS; 2015.

17. Meier GSO, Dellaroza MSG, Martins EAP. Indicadores pré-hospitalares na avaliação da qualidade da assistência ao paciente com síndrome coronariana aguda. Rev Gauch Enferm. 2015; 36(3):49-55.

18. Amaral CS, Reck AZC, Souza DS, Nuñez ARG, Blatt CR, Weis AH, et al. Situações de urgência e emergência na atenção primária reguladas pelo SAMU. J Health NPEPS. 2018; $3(1): 241-252$.

19. Wen TS. Acupuntura Classica Chinesa. São Paulo: Cultrix; 2014.

20. Odoul M. Diga-me onde dói que eu ti direi por que. São Paulo: Elsevier; 2003.

21. Messa JBL, Garme-Leiza JR, AguilarGarcia MD, López CA, Fernández JA, et al. Factores de riesgo cardiovascular en el ritmo circadiano del infarto agudo de miocárdio. Rev Esp Cardio. 2004; 57(9):850-858.

22. Poutriel KT, López AJR, Pestana EN, Martinéz FG. Ritmo circadiano en el infarto agudo del miocardio. AMC. 2009; 13(1). 
23. Cavalcante A, Santos ADAA, Braz DDS, Trindade LSD, Barros AMMS, Souza DS. Identificação precoce da síndrome coronariana aguda: uma revisão bibliográfica. Cad grad ciênc bio saúde. 2017; 4(2):218.
24. Nunes FMP, Silva AB. Assistência ao

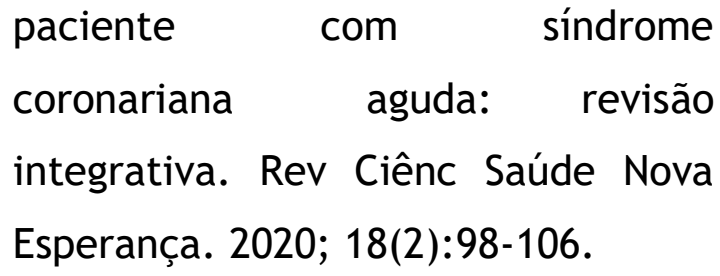

Financiamento: Os autores declaram que não houve financiamento.

Conflito de interesses: Os autores declaram não haver conflito de interesses.

\section{Participação dos autores:}

- Concepção: Martins EAP, Ribeiro BMSS, Moreira DV, Silverio-lopes S, Maier GSO.

- Desenvolvimento: Martins EAP, Ribeiro BMSS, Moreira DV, Silverio-lopes S, Maier GSO.

- Redação e revisão: Martins EAP, Ribeiro BMSS, Moreira DV, Silverio-lopes S, Maier GSO.

Como citar este artigo: Martins EAP, Ribeiro BMSS, Moreira DV, Silverio-lopes S, Maier GSO. Síndrome coronariana aguda associada a teoria do relógio biológico da medicina tradicional chinesa. J Health NPEPS. 2020; 5(2):337-350.

Submissão: $28 / 06 / 2020$

Aceito: $17 / 10 / 2020$

Publicado: 04/12/2020 